\title{
Identité et association en éducation relative à l'environnement
}

Edgar González-Gaudiano

\section{(2) OpenEdition}

Édition électronique

URL : http://journals.openedition.org/ere/6569

DOI : $10.4000 /$ ere.6569

ISSN : 2561-2271

Éditeur

Centr'ERE

Référence électronique

Edgar González-Gaudiano, « Identité et association en éducation relative à l'environnement », Éducation relative à l'environnement [En ligne], Volume 3| 2002, mis en ligne le 20 mars 2002, consulté le 16 avril 2021. URL : http://journals.openedition.org/ere/6569; DOI : https://doi.org/10.4000/ere. 6569

Ce document a été généré automatiquement le 16 avril 2021. 


\title{
Identité et association en éducation relative à l'environnement
}

\author{
Edgar González-Gaudiano
}

1 Le concept d'identité se voit attribuer différentes significations. Bien que les diverses approches de ce concept complexe aient été remises en question par plusieurs auteurs, principalement par les maîtres du doute - Nietzsche, Heidegger et Freud - et plus tard par d'autres comme Foucault, Wittgenstein, Lacan, Derrida et Laclau, pour ne mentionner que quelques-uns des plus connus, il m'apparaît important de revoir certaines de ses caractéristiques en les appliquant au domaine de l'éducation relative à l'environnement (ERE). Nous verrons également comment la problématique de l'identité de l'ERE et celle des acteurs du domaine est liée à son fort potentiel d'association ${ }^{1}$ : liens avec des domaines connexes, collaborations, coopérations, réseaux, etc.

\section{Problématique de l'identité de l'ERE}

2 Lorsqu'il est question d'éducation relative à l'environnement, nous admettons volontiers qu'il s'agit d'un domaine polyphonique, aux facettes multiples. Cette polyphonie rassemble une grande quantité de voix provenant d'aires disciplinaires des plus diversifiées, qui analysent les réalités à partir des approches théoricométhodologiques qui leur sont propres. Le dialogue entre ces différentes voix représente l'un des principaux défis du domaine de l'ERE; c'est d'ailleurs également le cas pour les différents champs d'intégration disciplinaire contemporains.

Mais cette polyphonie de l'ERE ne se limite certes pas au seul domaine scientifique. Je fais référence ici à la myriade de projets, de propositions et de discours impliquant diverses visions du monde, des plus poétiques au plus catastrophistes. Comme il s'agit d'un domaine en élaboration, nous nous trouvons en terrain mouvant, instable, ouvert et caractérisé par des différences marquées entre les pays et les régions, entre les secteurs et les groupes professionnels. La diversité est donc l'une des particularités 
intrinsèques du domaine de l'éducation relative à l'environnement, ce qui en fait un univers hétérogène et complexe ${ }^{2}$, sans identité unique ou permanente.

En tant que construction sociale située en contexte historique, l'éducation relative à l'environnement apparaît comme une manière de "diaspora" de discours sur l'environnement et l'éducation. Il arrive aussi fréquemment qu'elle présente différents types de discours hybrides; certains d'entre eux intègrent à des propositions pédagogiques scolaires plus conventionnelles les perspectives institutionnelles "officielles» relatives à la problématique environnementale, alors que d'autres répondent à des approches critiques plus ou moins radicales donnant lieu à des projets alternatifs ou marginaux (pour ne mentionner que deux cas extrêmes, rarement présents à « l'état pur»).

5 En ce sens, on peut considérer que le domaine de l'ERE s'étend d'un «centre» (conservateur) à une "périphérie » (critique et innovante), bien que de nombreux éducateurs environnementaux n'arrivent pas à distinguer les différents discours, et encore moins leurs particularités.

6 Le domaine de l'éducation relative à l'environnement a généralement été dominé jusqu'ici par le discours institutionnel officiel de l'UNESCO dans le cadre du Programme international d'éducation relative à l'environnement. Ce n'est que récemment que ce "centre " s'est déplacé vers la proposition de l'éducation pour un développement durable, d'abord appuyée par le projet PED (Population, Environnement et Développement) puis par le Programme d'Éducation pour un avenir viable. En réaction, la "périphérie " s'est également reconfigurée et son identité, quoique précaire, a donné naissance à de nouvelles voix discordantes se portant à la défense d'un domaine - celui de l'éducation relative à l'environnement - qui, au cours des dernières décennies, avait pourtant réussi à obtenir son " certificat de citoyenneté ».

7 Ces différents niveaux d'identification et d'appartenance, dans lesquels s'articule la complexe structure de l'identité des éducateurs environnementaux, correspondent à de multiples codes culturels en vigueur au sein du domaine de l'ERE. C'est selon ces codes qu'a été interprété et évalué, de diverses façons, le changement de discours institutionnel de l'UNESCO, provoquant de nouveaux antagonismes et de nouvelles différences. Le domaine de l'ERE traverse actuellement une période d'instabilité si particulière qu'il s'est même délivré son propre «acte de décès » lors de la Conférence internationale de Thessalonique (1997) ${ }^{4}$, ce qui n'a pas manqué de susciter de vives controverses. C'est ainsi que nous faisons face à de nouvelles positions ou « subjectivités » au sein de l'ERE, qui correspondent à autant de façons d'expérimenter le monde, à différentes formes d'identité et d'appartenance qui proposent une redéfinition des objectifs, des alliances, des accords et des résultats attendus.

\section{Un potentiel d'association}

8 Les constats précédents sur l'identité du domaine de l'ERE nous permettent d'aborder, quoique succinctement, les différentes possibilités d'association (liens, collaborations, coopérations, réseaux, etc.). En effet, en tant que domaine polyphonique comportant de multiples facettes, l'éducation relative à l'environnement possède un grand potentiel pour l'« associationnisme ». Cette particularité n'exclut cependant pas la possibilité de conflits entre les parties, puisqu'il est question d'un domaine plus ou moins structuré, 
qui se distingue par le caractère relationnel d'une identité sociale très diversifiée et qui n'échappe pas bien entendu aux relations de pouvoir.

9 Afin d'analyser les possibilités d'association entre des personnes issues de divers domaines disciplinaires ou entre différents types d'organisations, il importe de prendre en considération les liens qui existent entre les processus identitaires inhérents aux diverses disciplines et leur univers symbolique, et de tenir compte des nombreuses dimensions qui entrent dans la structure complexe de la société. Les disciplines scientifiques, aussi bien que les professions connexes, correspondent à des contextes normatifs et régulateurs des activités discursives 5 . En s'intégrant au plan social (dont elles ne sont pas isolées, rappelons-le), ces activités discursives s'inscrivent également aux niveaux symbolique et culturel.

10 À cet égard, il important de considérer les processus d'institutionnalisation et de professionnalisation comme des axes permettant de réactiver et de renforcer les vecteurs d'appartenance dans la configuration d'un domaine - comme celui de l'ERE dont la structure est encore précaire. De tels processus offrent un grand potentiel d'associations diverses, entre autres parce que l'admission aux programmes académiques ne dépend pas exclusivement d'un profil professionnel ou occupationnel déterminé.

11 La professionnalisation ${ }^{6}$ est étroitement liée au processus d'institutionnalisation, laquelle implique l'interaction de sujets occupant différentes positions de pouvoir ainsi que la consolidation de patrons normatifs, de modèles d'organisation, de schémas régulateurs d'échange aussi bien que de valeurs sociales et culturelles. L'institutionnalisation (dont celle de l'éducation relative à l'environnement) suppose donc, pour le meilleur et pour le pire, un certain contrôle de positions, contrôle basé sur des critères implicites ou explicites d'inclusion ou d'exclusion.

12 Les identités et les pratiques discursives concernées ont un caractère tout à fait historique et le cadre de référence disciplinaire de même que le mode d'institutionnalisation occupent une place importante dans les règles de comportement académique, engendrant entre autres des modèles ou des rites d'initiation spécifiques. Ces éléments sont déterminants quant aux possibilités et aux stratégies d'association dans un domaine comme celui de l'ERE ; en effet, les expériences qui négligent de définir les objectifs et les résultats attendus sont généralement à la merci d'autres intérêts qui tirent profit de ce laxisme.

13 Par ailleurs, comme l'éducation relative à l'environnement a été élaborée aux limites du social tout autant que dans ses interstices, elle peut facilement établir des liens avec d'autres domaines émergents et périphériques de l'éducation comme l'éducation pour les droits humains, pour la démocratie, pour la paix, etc. L'ERE étant un domaine en évolution, on y remarque à quel point ce qui la constitue prédomine sur ce qui est déjà constitué. C'est une structure précaire, instable, constamment disloquée et soumise à l'implacable aléatoire ; pour cette raison et justement à cause de ces particularités, cette structure présente une configuration qui réaffirme son caractère d'ouverture, lui conférant la possibilité d'établir d'innombrables articulations, impensables dans d'autres domaines en émergence. C'est bien là que réside son formidable potentiel d'association.

14 L'éducation relative à l'environnement est donc investie d'un important potentiel articulatoire et, par conséquent, d'une grande capacité participative, puisque ses 
objectifs et ses caractéristiques lui permettent de créer divers types de liens (conceptuels, stratégiques, professionnels, institutionnels ou autres). Ceci peut facilement se vérifier dans les réseaux d'éducateurs environnementaux du Mexique, par exemple, qui rassemblent des personnes exerçant des professions et des occupations diverses, provenant de plusieurs secteurs institutionnels et travaillant dans des conditions variables, avec des groupes très différents. Par ailleurs, ces réseaux ont créé des alliances avec d'autres groupes, comme par exemple des organisations de consommateurs dont les objectifs, quoiqu'en lien, n'impliquaient pas à priori de thématiques environnementales. Enfin, ces réseaux ont également créé des liens avec d'autres secteurs éducatifs en émergence comme celui l'éducation en matière de population, l'éducation pour la santé et l'éducation pour les droits humains ; et se sont ralliés à des luttes connexes comme celles de la condition féminine, de la paix, de la transition démocratique, des personnes handicapées, sans oublier la plus importante de toutes, le combat contre la pauvreté.

Comme nous l'avons vu précédemment, le domaine de l'éducation relative à l'environnement s'inscrit dans différents espaces et projets que nous pourrions qualifier de "socio-environnementaux ", soulignant ainsi le caractère global de cette dimension de l'éducation. Les propositions trop axées sur le " côté vert » de l'éducation relative à l'environnement se vident donc de leur sens, dans leur nostalgique tentative de se réapproprier les approches naturalistes du XIXème siècle impliquant une stratégie de conservation qui, de par ses caractéristiques, est vouée à l'échec, surtout dans les pays en voie de développement.

Le domaine reste à consolider et à enrichir par des associations avec différents champs et acteurs. Les possibilités de renforcer ce potentiel d'association sont à leur tour liées aux possibilités accrues de communication ; ce qui exige de la part des éducateurs environnementaux un effort de formation beaucoup plus important que celui généralement observé jusqu'à présent.

Il serait par ailleurs important de se pencher sur la formation des enseignants du primaire dont le travail est essentiel. Malheureusement, ces derniers limitent trop souvent leur travail à la poursuite des objectifs du curriculum scolaire et à l'adoption d'approches traditionnelles. Leur formation se résume le plus souvent à des contenus et à des techniques didactiques spécifiquement destinés au niveau scolaire concerné. On observe que le lien entre l'éducation relative à l'environnement et l'école primaire n'a pas du tout favorisé les articulations évoquées précédemment et la composante " verte » $\mathrm{y}$ reste donc fortement dominante.

18 Cependant, certaines expériences en milieu scolaire, surtout celles liées au milieu rural impliquant une population culturellement différente, et d'autres, d'origine urbaine, aux intérêts plus intégrateurs, démontrent non seulement que l'association à d'autres luttes s'avère possible, mais qu'elle constitue une stratégie pédagogique plus riche, permettant aux activités scolaires de donner un sens nouveau à la vie des élèves et des enseignants.

19 Le présent, tout comme l'avenir de l'ERE, sont influencés par sa capacité naturelle d'association. Les nouvelles conditions de communication ont favorisé l'échange d'expériences et de projets et, par la même occasion, ont enrichi un terrain déjà fertile en rencontres et en convergences. Cet état de fait a, entre autres, été démontré lors de récentes expériences sur Internet comme le On Line Colloquium, organisé par le Canadian Journal of Environmental Education, ou encore le Symposium orchestré par des 
organisations néerlandaises. Ces événements ont permis à de nombreuses voix provenant de différentes parties du globe de se faire entendre, alors que sans les nouvelles possibilités technologiques de communication, ces échanges auraient été infiniment plus difficiles. Tout ceci continuera sans doute de se manifester par le biais d'organisations de plus en plus nombreuses, complexes, sur les terrains de pratique comme dans l'espace virtuel.

\section{BIBLIOGRAPHIE}

González Gaudiano, E. (2000). La complexité en éducation relative à l'environnement. Tópicos en educación ambiental, 2(4), 21-32.

González Gaudiano, E. (1998). Centre et périphérie en éducation relative à l'environnement. Un point de vue essentialiste. México : Mundi Prensa.

UNESCO. (1997). Éducation pour un développement durable : une vision transdisciplinaire en vue d'une action concertée. Paris : Unesco.

\section{NOTES}

1. Note de traduction : Le mot "partenariat" n'existe pas en langue espagnole, où le terme colaboración est le plus fréquemment utilisé pour désigner toute forme de liens entre différents acteurs ou organismes au sein de projets. Dans cet article, c'est le concept d' "association » (asociación) qui est retenu par l'auteur, car il recouvre une diversité de types de relations entre les champs de savoir et de pratique, et entre différents types d'acteurs. (Lucie Sauvé)

2. Concernant la complexité du domaine de l'ERE, voir González-Gaudiano (2000).

3. De telles voix discordantes se sont exprimées en différents lieux comme par exemple dans le Canadian Journal of Environmental Education (CJEE), plus particulièrement lors du On Line Colloquium qui s'est tenu en 1999, ou encore dans le Environmental Educator, bulletin publié par la North American Association for Environmental Education (NAAEE).

4. L'acte de décès a été émis dans le document de base de la Conférence internationale de Thessalonique (Grèce) en 1997 (UNESCO, 1997).

5. Par «activités discursives", nous entendons tous les actes linguistiques et extralinguistiques qui leur donnent sens.

6. Nous devons reconnaître que le concept de professionnalisation a une origine aristocratique liée à l'apparition des professions libérales au XIXème siècle, professions libérales qui, à leur tour, ont donné naissance aux corporations, aux académies, aux ordres professionnels, etc. En ce qui à trait à la professionnalisation en éducation relative à l'environnement dans le cas du Mexique et de l'Amérique latine, voir González Gaudiano (1998). 


\section{AUTEUR}

\section{EDGAR GONZÁLEZ-GAUDIANO}

Edgar J. González-Gaudiano est professeur à la Universidad Nacional Autónoma de México et conseiller du Secrétariat d'Éducation publique (Mexique) sur les questions d'éducation relative à l'environnement et au développement durable. Il est également éditeur de la revue ibéroaméricaine Tópicos en Educación Ambiental et vice-président de l'Académie nationale d'éducation relative à l'environnement, une ONG visant à promouvoir la formation et la recherche en éducation relative à l'environnement. Edgar G. Gaudiano s'intéresse entre autres à l'analyse critique du discours environnemental en Amérique latine et dans les Caraibes. 